Egyptian

Orthodontic Journal

\title{
IMPLANT VERSUS NANCE HOLDING ARCH ANCHORGE DURING MAXILLARY CANINE RETRACTION
}

\author{
Maher A. Fouda ${ }^{1}$, Fouad Al-Belasy ${ }^{2}$, Shaza H.Abd El-Nabi ${ }^{3}$ \\ Marwa S. Shamaa ${ }^{4}$
}

\section{ABSTRACT:}

The aim of the present work is to determine the anchorage potential of titanium microimplant for maxillary canine retraction and to measure the anchorage loss using microscrew implants compared to Nance holding arch as intraoral anchorage during maxillary canine retraction in Angle's class I crowding. Sample of this study consisted of 10 females ranging in age from 18-24 years. 5 received micro-screw implants as a mean of anchorage and the remaining 5 received conventional molar anchorage as $\mathcal{N a n c e}$ holding arch. The molar anchorage loss was determined in both the implant anchored cases and the molar anchored cases by superimposing the lateral cephalometric tracings before and after canine retraction along the palatal plane registered at the anterior nasal spine. The horizontal distances from the pterygoid vertical to the distal surfaces of the first molars were calculated to measure the anchorage loss. In present study all implants remained stable throughout the treatment period and no damage was registered in any implant under the condition of implant loading. Also, no implant deformation was detected in any implant., anchorage loss was detected more significantly $(P<.0007)$ in patients with

1- Prof. of Orthodontics Faculty of Dentistry, Mansoura University.

2- Prof. of Oral Surgery Faculty of Dentistry, Mansoura University.

3- Lecturer of Orthodontics Faculty of Dentistry, Mansoura University.

4 - Demonstrator of Orthodontics Faculty of Dentistry, Mansoura University. 
intraoral anchorage through the Nance holding arch compared to patients with anchorage through orthodontic mini-implants 6oth on the right and left side. In the $\mathcal{N a n c e}$ group the mean anchorage Coss was $1.4 \mathrm{~mm}( \pm 0.418)$ on the right side and $1.42 \mathrm{~mm}( \pm 0.437)$ on the left side. On the other hand no molar rotation was detected in the present study either by using the Nance holding arch or the microimplant. So, this study is a further prove of the beneficial use of microimplants as a good anchorage method with profound control of molar rotation.

\section{INTRODUCTION}

Anchorage in orthodontics is the most important factor that determines the treatment and result. ${ }^{1}$ Salzmann $(1966)^{2}$, stated that "regardless of the skill one may possess in the space closure following the extraction of the teeth, the teeth in the posterior buccal segment will be displaced mesially to some extent".

If skeletal anchorage could be applied to orthodontic tooth movement it might offer capabilities therefore unavailable. The need for extraoral forces and the removal of teeth might be greatly reduced. Extraoral anchorage has severe limitations because it requires excellent patient cooperation $^{3}$.

Tooth borne anchorage is one of the greatest limitations of modern orthodontic treatment, because teeth move in response to forces. While extraoral anchorage can be used to supplement tooth borne anchorage and to deliver forces in directions not possible with intraoral force ${ }^{3}$.

Various techniques to reinforce anchorage have been devised and used in orthodontic practice. However, even some of the best known intraoral appliances (palatal or lingual bars, the Nance holding arch and intermaxillary elastics) have undesirable side effects, including protrusion, extrusion and tipping of some teeth ${ }^{1}$.

There is no question that dental implants have been the most influential change in dentistry since the last half of the 20th Century as they are well proven and highly useful. The mini-implant use as orthodontic anchorage has been recently reported in retraction of anterior 
teeth, namely the maxillary canines. This was based on the successful use of mini-implant as anchorage for extrusion of impacted teeth and correction of dental protrusion in preprothetic treatment ${ }^{4}$.

On the other hand implants are in direct contact with bone, possessing no periodontal ligament. As a result, they do not move when orthodontic/orthopedic force is applied and therefore can be used "absolute anchorage"

Advances in implant dentistry have made it possible to use implants for anchorage in adult orthodontic patients. After the introduction of implant, its use as a source of orthodontic anchorage was widely reported in literature ${ }^{1}$. The use of implant as anchorage for the retraction of canines is not a viable alternative to conventional molar anchorage. Orthodontic mini-implants (OMIs) are a predictable, effective and well tolerated in producing immediate orthodontic anchorage for adolescents. Neither the timing of force application nor the force itself precipitated failure of OMIs $^{6}$.The aims of this work were:

1- To determine the anchorage potential of titanium microscrew implants of maxillary canine retraction in Angle's class I crowding.

2- To measure anchorage loss using titanium microscrew implants compared to Nance holding arch as intraoral anchorage during maxillary canine retraction in Angle's class I crowding.

\section{MATERIAL AND METHODS}

Sample of this study consisted of 10 females ranging in age from 18-24 years. They were selected from the Outpatient Clinic of the Orthodontic Department, Faculty of Dentistry, Mansoura University. Of those 10 females, 5 received micro-screw implants as a mean of anchorage and the remaining 5 received conventional molar anchorage as Nance holding arch.

Each subject of the sample had to fulfill the followings: Class I Angle with crowded anterior teeth; Maximum anchorage requirements; no previous Orthodontic treatment and no abnormal oral habits.

All the patients were informed of the procedure and signed consent. 


\section{I-In the Implant group:}

\section{1- Implantation site and Aiming device:}

Before implantation of the miniscrews, the panoramic radiograph was checked visually to assess the parallelism of the roots and the availability of bone between the roots of the maxillary second premolars and the first molars. A piece of stainless steel $0.016 \mathrm{X} 0.022$ inch wire was shaped to encircle the prescribed site of implantation between the roots of the second premolar and the first molar within the attached gingival with one end inserted in the first molar tube and the other end tied to the second premolar bracket. Pariapical radiographs were taken with long cone $\mathrm{x}$ - ray machine and paralleling technique to assess the point of entry of the miniscrew in relation to the roots of the adjacent teeth and its position was modified accordingly ${ }^{7}$.

\section{2- Implantation Procedure:}

The implantation site between the roots of the second premolar and the first molar. The predetermined point of insertion was punched with a periodontal probe to make an indentation bleeding point. A number 2 rose head bur was mounted on low speed contra-angle handpiece to minimize the bone over heating and to be able to feel the transition from cortical bone to medullary bone. The hand piece was adjusted at angle of $30^{\circ}-60^{\circ}$ with the surface of bone directed apically, at the bleeding point. The rotatory bur was allowed to penetrate the soft tissue buccal cortical plate, with a very mild pressure, till transition from the cortical to the medullary bone was felt through the unrested progress of the drill ${ }^{8}$.

The miniscrews (Absoanchor Mini-implant (Dentos Inc.), that were $1.5 \mathrm{~mm}$ in diameter and $8 \mathrm{~mm}$ in length) in 4 patients and Aarhus system mini-implant (1.5 $\mathrm{mm}$ in diameter and $10.8 \mathrm{~mm}$ in length)in one patient) $\}$, were ejected from its sterile packaging loaded in the standard long - handle driver and the miniscrew was self drilled into the bone through the pilot hole. The miniscrews were screwed into bone till all of the threaded section got engaged into the bone leaving the head of miniscrew exposed into the oral cavity (open technique) for ligation of the orthodontic attachment ${ }^{8}$. After implantation of the miniscrews; extractions of the maxillary first premolars were done.

\section{3- After implant placement:}

Twist - flex stainless steel arch wires of $0.0150 "$ and $0.0175 "$ were used for alignment of the mesialy inclined maxillary canines in some 
Egyptian

Orthodontic Journal

cases. A plastic sleeve tube was cut and put in the incisal area in cases of crowded upper incisors (i.e incisors were not tied to the arch wire). Then after uprighting the canines: stainless steel arch wires of "0.012", "0.014", "0.016" and "0.018" were used consequently to align the canines.

II_Convential molar anchorage group:

Fabrication of the Nance holding arch:

The first permanent molars were banded and single buccal tubes with books were welded to the buccal surfaces of the bands. The bands with buccal tubes remained on the teeth and the Nance holding arch was fabricated by taking an alginate impression with the first permanent molar bands in their place. After setting of the alginate; the impression was removed from the patient mouth and the molar bands were fitted in their place in the alginate impression. Then, the impression was poured in dental plaster. Nance holding arch was constructed in $0.9 \mathrm{~mm}$ hard stainless steel wire and cemented on the first permanent molars inside the patient's mouth. The first bicuspids were extracted in two visits with a week interval between the visits

\section{III-Maxillary canine retraction:}

In the implant group the maxillary canines were retracted using $0.016 \times 0.022$ inch stainless steel arch wire with the Nickel Titanium closed - coil springs stretched between the implant head and the distal wing of the canine bracket, in both sides of the maxillary arch. While in the second group the maxillary canines were retracted using 0.016 " x 0.022 " stainless steel arch wire with the Nickel - Titanium closed - coil springs stretched between the hook on the buccal surfaces of the molar bands and the distal wing of the canine brackets in both sides of the maxillary arch. In both groups, the Nickel-Titanium coil spring delivered a force of $100 \mathrm{gm}$ measured clinically by a stress and tension gauge. The molar anchorage loss was determined in both the implant anchored cases and the molar anchored cases by superimposing the lateral cephalometric tracings before and after canine retraction along the palatal plane registered at the anterior nasal spine. The horizontal distance from the pterygoid vertical to the distal surfaces of the first molars were calculated to measure the anchorage loss.

Dental casts were also utilized to assess the dental arch changes before and after completion of canine retraction. The pre and post retraction upper models were photocopied on a cannon machine by a 1 to 1 magnification. A flu-master pen was used to erect points on the mesial, 
distal corners of the maxillary canines on the apex of the palatal interdental papilla between the upper central incisors and tips of the four cusps of the maxillary first molars. A line was drawn through the apex of the palatal interdental papilla between the central incisors perpendicular to the heel of the upper cast representing the median maxillary line (MML). The following measurements were assessed:1-Angle of rotation of the maxillary canines (AC), 2-Distal movement of the canine (DC), 3-Angle of rotation of maxillary first molars (AM), 4-Mesial movement of the maxillary first molars (MM). All measurements were taken with a Boley gauge to the nearest tenth of a millimeter.

\section{RESULTS}

Table (1) shows that there was significantly less anchorage loss in the implant group compared to the Nance group on both sides $(\mathrm{p}<0.0007)$. In Table (2), the cephalometric and cast measurements of the patients enrolled in this study were tabulated. The variables were tested by paired $t$ test for equal variance with level of significance $\mathrm{P}<0.05$. A significant decrease of $\mathrm{AC}(\mathrm{L})$ angle, $\mathrm{MM}(\mathrm{L})$ and $\mathrm{MM}(\mathrm{R})$ distances and increase in DC $(\mathrm{L})$ and $\mathrm{DC}(\mathrm{R})$ distance were detected in the Nance group after canine retraction compared to the corresponding initial values before treatment. While in the implant group the significant changes following treatment were an increase in DC (L) and DC(R) distances.

Table (1): Mean Anchorage Loss in Implant and Nance groups.

\begin{tabular}{|ll|l|}
\hline \multicolumn{1}{|c|}{ Implant patients } & Nance patients \\
\hline Rt & \\
\hline Mean $(\mathrm{mm})$ & 0.2 & 1.4 \\
\hline S.D & 0.274 & 0.418 \\
\hline S E of mean & 0.123 & 0.187 \\
\hline P & & \\
\hline Lt & $0.007^{*}$ \\
\hline Mean $(\mathrm{mm})$ & 0.2 & 1.42 \\
\hline S.D & 0.283 & 0.427 \\
\hline S E of mean & 0.127 & 0.191 \\
\hline P & \multicolumn{2}{|l}{} \\
\hline
\end{tabular}

* significant at $\mathrm{P}<0.05$. 
Table (2): Comparison of Cepalometric and cast measurements of Nance and implants groups, before and after canine retraction (Angles in degrees,distance in $\mathrm{mm}$ ).

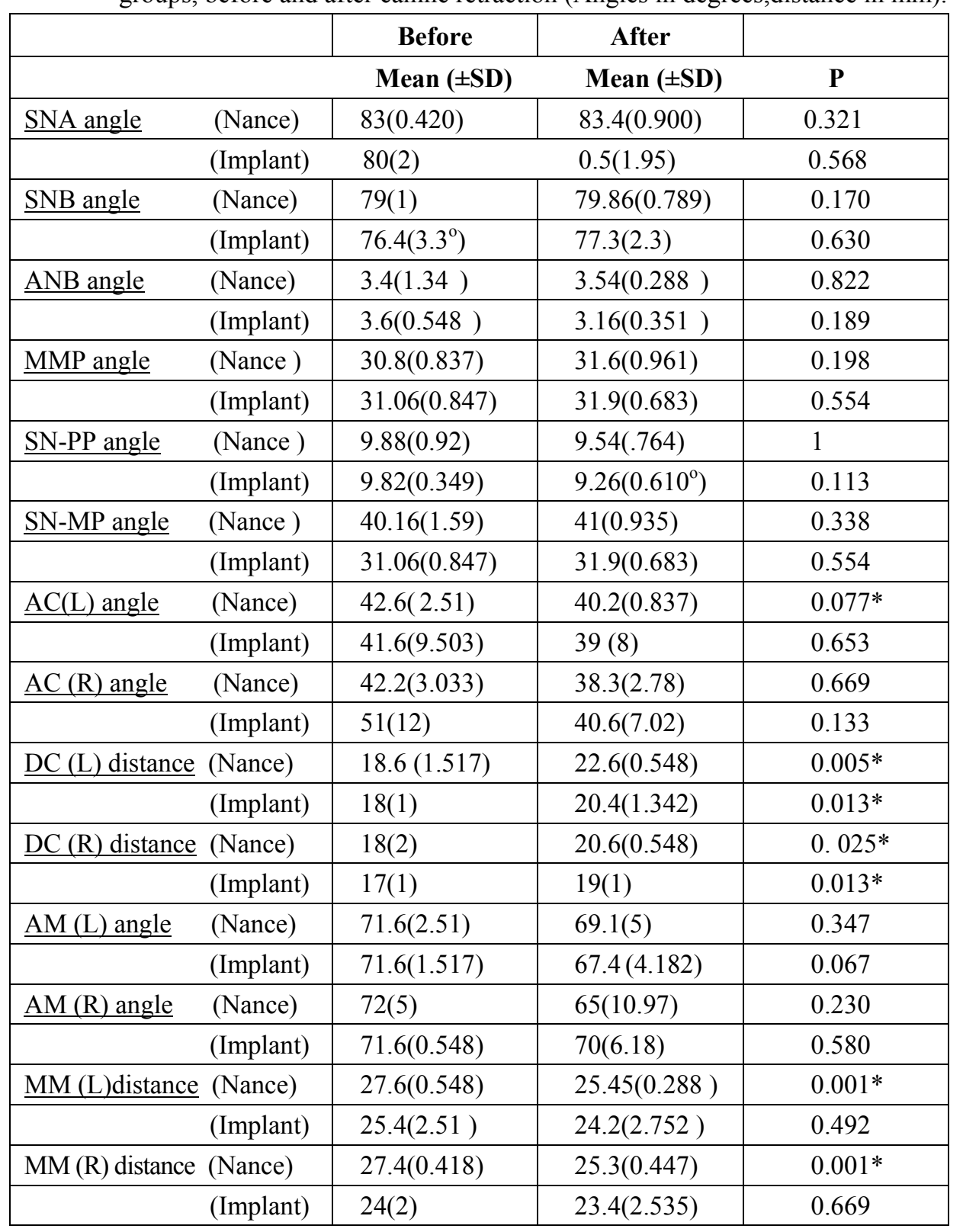

* Significant at $\mathrm{P}<0.05$. 
Table (3): Unwanted effects in implant patients (10 implant in 5 patients):

\begin{tabular}{ll} 
Implant damage & $0 / 10$ \\
Implant deformity & $0 / 10$ \\
Peri-implant inflammation & $4 / 10$ \\
\hline Complications during insertion & $1 / 10$ \\
Lack of stability & $0 / 10$ \\
Faulty insertion & \\
\hline Complications during loading & $2 / 10$ \\
Mucosal hypertrophy & \\
\hline & \\
Table (4): Unwanted effects in Nance patients $(5):$ & $1 / 5$ \\
\hline & $5 / 5$ \\
\hline
\end{tabular}

Tables (3) and (4) show the unwanted effects of the implants and the Nance holding arch.

(Fig.1): Mini-screw implant Case:

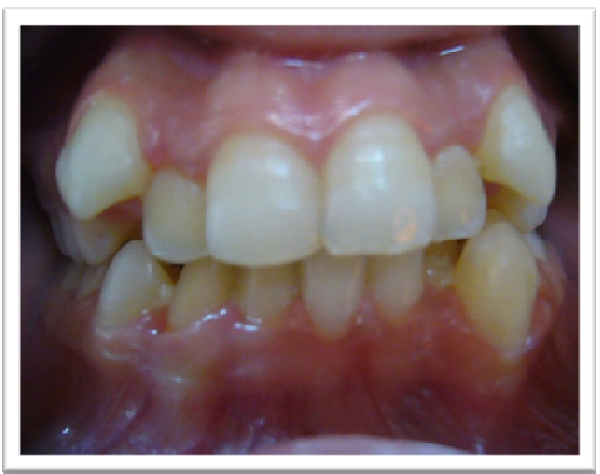

Before treatment

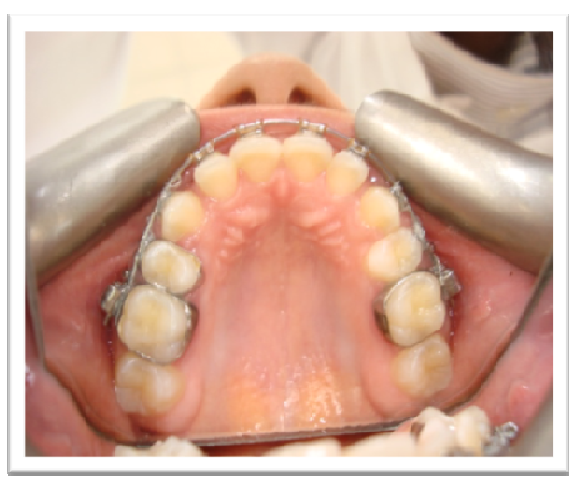

The canines were retracted successfully 
(Fig.2): Nance holding arch case:

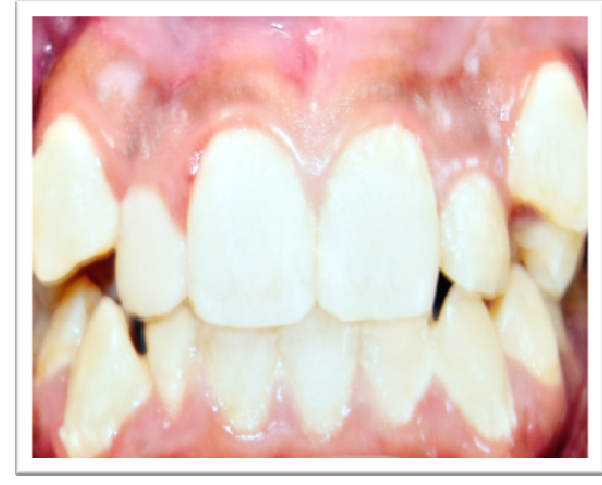

Before treatment

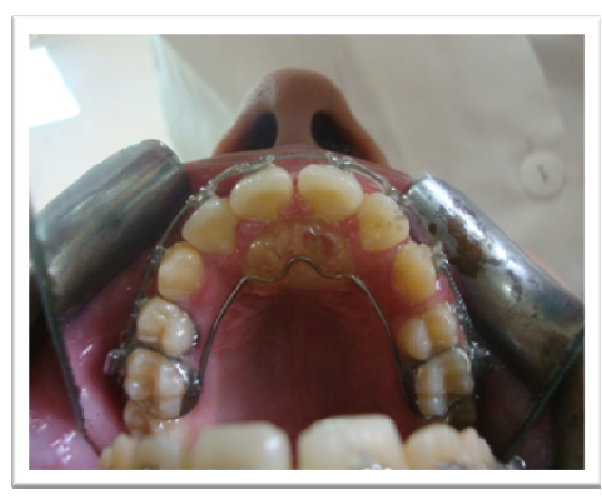

The canines were retracted successfully

\section{DISCUSSION}

Tooth borne anchorage is one of the greatest limitations of modern orthodontic treatment, because teeth move in response to forces. While extraoral anchorage can be used to supplement tooth borne anchorage and to deliver forces in directions not possible with intraoral forces. Extraoral anchorage has severe limitations because it requires excellent patient cooperation $^{3}$.

Today, anchorage control is a major concern in the design of orthodontic appliances .Various techniques to reinforce anchorage have been devised and used in orthodontic practice. However, even some of the best known intraoral appliances (palatal or lingual bars, the Nance holding arch and intermaxillary elastics) have undesirable side effects, including protrusion, extrusion, extrusion, and tipping of some teeth ${ }^{1}$.

After the introduction of implant, its use as a source of orthodontic anchorage was widely reported in literature ${ }^{1}$. Specifically designed orthodontic implants have been placed in various locations such as the alveolar bone ${ }^{9}$, the retromolar region ${ }^{10}$, the midpalatal region ${ }^{11}$, and the lingual ${ }^{12}$ and buccal ${ }^{13}$ Cortical plates. 
The use of implant as anchorage for the retraction of canines is a viable alternative to conventional molar anchorage. Orthodontic mini-implants (OMIs) are a predictable, effective and well tolerated in producing immediate orthodontic anchorage for adolescents. Neither the timing of force application nor the force itself precipitated failure of OMIs ${ }^{6}$.

Only relatively few studies have measured the amount of anchorage loss during canine retraction in humans, and that at that date, there were no studies measuring anchorage loss with implant assisted canine retraction. In his study, an attempt was made to evaluate the anchorage loss by using more than one variable: by superimposing and by measuring the amount of anchor loss (L-shaped wires as reference points) in lateral cephalogram before and after retracation ${ }^{1}$.

The aim of the present work is to determine the anchorage potential of titanium microimplant for maxillary canine retraction and to measure anchorage loss using microscrew implants compared to Nance holding arch as intraoral anchorage during maxillary canine retraction in Angle's class I crowding. Sample of this study consisted of 10 females ranging in age from 18-24 years. They were selected form the Outpatient Clinic of the Orthodontic Department, Faculty of Dentistry, Mansoura University. Of those 10 females, 5 received micro-screw implants as a mean of anchorage and the remaining 5 received conventional molar anchorage as Nance holding arch. In present study, anchorage loss was detected more significantly $(\mathrm{P}<.0007)$ in patients with intraoral anchorage through the Nance holding arch compared to patients with anchorage through orthodontic mini-implants both on the right and left side. In the first group the mean anchorage loss was $1.4 \mathrm{~mm}( \pm 0.418)$ on the right side and 1.42 $\mathrm{mm}( \pm 0.437)$ on the left side. Nearly similar results were previously reported by several investigators ${ }^{14,15}$. In both: Eid, $1988^{14}$ and Fouda, $2006^{15}$ studies, the anchorage loss was more significant on the left than on the right, while in the present study the difference is insignificant between both sides.

(El-Dakroury et al, 1996) ${ }^{16}$, compared the Nance Holding arch with 2 other intraoral anchorage arches ( $\mathrm{H}$ bar and Transpalatal), they recommended the use of Nance holding arch for intraoral anchorage. But 
Egyptian

Orthodontic Journal

the present work support the use of mini-implant anchorage because of the minimal anchorage loss detected $[0.2 \mathrm{~mm}( \pm 0.238)$ and $0.2 \mathrm{~mm}$ $( \pm 0.274)$ in left and right sides respectively]. This is in agreement with the finding of Thiruvenkatachari et $\mathrm{al}^{1}$, they achieved a successful canine retraction without any anchorage loss in the implant side compared to a mean loss of $1.6 \mathrm{~mm}$ on the molar anchorage side. On the other hand no molar rotation was detected in the present study either by using the Nance holding arch or the microimplant. So, this study is a further prove of the beneficial use of microimplants as a good anchorage method with profound control of molar rotation.

In the present work no significant differences was detected before compared to after canine retraction in both groups regarding MMP, SN-PP and SN-MP indicating no deleterious effect on the lower face height.

In the present study all implants remained stable throughout the treatment period and no damage was registered in any implant under the condition of implant loading. Also, no implant deformation was detected in any implant. Peri-implant inflammation was detected around 4 out of the 10 implants used this is most probably related to improper oral hygiene, because this inflammation subsided rapidly with proper oral hygiene measures. On the other hand food accumulation under the acrylic bottom was detected in all cases anchored by the Nance holding arch and severe mucositis related to improper oral hygiene was detected in one case. Therefore microimplants are successful as a rigid anchoring system with minimal side effects.

\section{CONCLUSIONS}

In the proper patient and implant selection, implants as anchorage for retraction of canines can be incorporated into orthodontic practices with complete success. Evaluation of the relationship of the insertion pathway with adjacent structures is needed to avoid iatrogenic damage. The minimal degree of anchorage loss that might be encountered can be prevented by proper training; however combined use of microimplants and other intraoral anchorage systems to reinforce anchorage may be of value for complete anchorage success. 
Egyptian

Orthodontic Journal

\section{REFERENCES}

1- Thiruvenkatachari B, Pavithranand A, Rajasigamani K and Kyung H M. (2006): Comparison and measurement of the amount of anchorage loss of the molar with and without the use of implant anchorage during canine retraction. AMJ orthod dent facial orthop, 129(4): 551-554.

2- Salzmann J A. (1966): Orthodontics under medicare and medicaid. Am J Orthod.: 922-926.

3- Creekmore T D and Eklund M K. (1983): The possibility of skeletal anchorage. J. clin . Orthod, 17: 266-269

4- Christensen G J (2006): The mini implant has arrived. J Am Dent Assoc. 137(3):387-390.

5- Rungcharassaeng K, Kanjy and Carsuso J M. (2005): Implants as absolute anchorage. J Calif dent Assoc, 33 (11) 881-888.

6- Garfinkle J S, Cunningham L L Jr, Beeman C S, Kluemper G T, Hicks E P, Kim M O. (2008): Evaluation of orthodontic mini-implant anchorage in premolar extraction therapy in adolescents. Am J Orthod Dentofacial Orthop. 133(5):642-653

7- Melsen B and Verna C. (2005): Minicrew implants the Aarhus anchorage system, Semin orthod, 11:24-31.

8- Sung J H, Kyung H M, Bae S M, Pank H S and Kwan. (2008): Microimplants in orthodontics. Publication of the dept of orthodontics, school of dentistry, Kyung pook National university, South Korea www. dentos co. kr.

9- Odman J, Lekholm U, Jemt T, Thilander B. (1997): Osseointegrated implants as orthodontic anchorage in the treatment of partially edentulous adult patients. Eur J Orthod, 31: 763-767.

10- Roberts W E, smith R k, Zilberman Y. (1984): Osseoss adaptation to continuous loading of rigid and osseous implants. J. ortho. Dentofac. Orthop, 86: 95-111.

11- Block M S and Hoffman D R. (1995): A new device of absolute anchorage for orthodontics. Am j. orthod. dentofacial orthop, 107: 251-258. 
12- Lee S T, Pank H S and Kyung H M. (2001): Micro-implant anchorage for lingual treatment of a skeletal class II malocclusion. J. clin Orthod, 35: 643-647.

13- Kyung S H, Choi J H, Park Y C. (2003): Miniscrew anchorage used to protract lower second molars into first molar extraction sites. J Clin Orthod., 37(10):575-579.

14- Eid F H. (1988): A comparative study to reveal the effect of different orthodontic force magnitude during canine retraction PH D Thesis, Faculty of Oral and Dental Medicine Cairo University.

15- Fouda A M. (2006): Dento-skeletal effects of anchorage loss during maxillary canine retraction. M.Sc. Thesis, Faculty of Oral Medicine, Mansoura University.

16- El -Dakroury A E, Labib A A and El -Kadi A A. (1996): Molar stability using different modalities of internal anchorage. Egyptian Orthod. J., 10: 47-56. 